Proceedings of the 43rd "Jaszowiec", International School and Conference on the Physics of Semiconductors, Wisła 2014

\title{
Peculiarities of Chemical Bonding in Crystals of the In-Se System
}

\author{
L.Yu. Kharkhalis ${ }^{a}$, K.E. Glukhov ${ }^{a}$ And M. SznAJder ${ }^{b, *}$ \\ ${ }^{a}$ Institute of Physics and Chemistry of Solid State, Uzhgorod National University, \\ 54 Voloshin St., 88000 Uzhgorod, Ukraine \\ ${ }^{b}$ Faculty of Mathematics and Natural Sciences, University of Rzeszów, S. Pigonia 1, 35-959 Rzeszów, Poland
}

\begin{abstract}
The investigation of the $a b$ initio band structure and the resulting spatial electron density distribution of the In-Se system in the framework of the density functional theory and the elementary energy bands concept is presented. It gives us reliable information about the valence band structure and peculiarities of the chemical bonding in these crystals. Some regularities in the evolution of the elementary energy bands topology is established, together with the choice of the actual Wyckoff position that is responsible for this topology and, at the same time, for the valence band formation in the $\mathrm{InSe}, \mathrm{In}_{2} \mathrm{Se}_{3}, \mathrm{In}_{4} \mathrm{Se}_{3}$, and $\mathrm{In}_{6} \mathrm{Se}_{7}$ crystals. The calculated Mulliken charges and the degree of ionicity allow to estimate the character of chemical bonding in these crystals.
\end{abstract}

DOI: 10.12693/APhysPolA.126.1146

PACS: 71.15.Mb, 61.50.Ah, 71.20.Nr

\section{Introduction}

After the discovery of excellent thermoelectric properties of the single crystalline rhombic semiconductor $\mathrm{In}_{4} \mathrm{Se}_{3}$ that are quantified by the dimensionless figure of merit, ZT, that appeared to be larger than 1.4 [1], an intensive research of the indium selenide group has started. The phase diagram of the In-Se system shows the existence of $\mathrm{InSe}, \mathrm{In}_{2} \mathrm{Se}_{3}, \mathrm{In}_{4} \mathrm{Se}_{3}$, and $\mathrm{In}_{6} \mathrm{Se}_{7}$ layered crystals. With the increase of the indium content in the compounds of the system, the decrease in their symmetry is observed, together with more complex chemical bonding in these compounds. By analyzing the interatomic distances in crystals of the In-Se system, it has been found that a different atomic environment for indium takes place e.g., the $\left(\operatorname{In}_{3}\right)^{5+}$ cluster in the $\mathrm{In}_{4} \mathrm{Se}_{3}$ crystal [2] and the dumbbell-like group of $\left(\operatorname{In}_{2}\right)^{6+}$ in the $\beta$ InSe [3]. A complicated atomic environment (tetrahedral and octahedral configurations) for indium and selenium atoms in $\mathrm{In}_{2} \mathrm{Se}_{3}$ and $\mathrm{In}_{6} \mathrm{Se}_{7}$ crystals is also observed [4, 5]. The average atomic distance $\operatorname{In}-\mathrm{Se}(\approx 2.5-3 \AA)$ for all compounds of the group is almost exactly the sum of the covalent radii, and the bonding between them is considered to be mainly covalent. In this paper we perform the studies on peculiarities of the chemical bonding for indium selenides from the viewpoint of electronic structure, using the elementary energy bands concept $[6,7]$ and the density functional theory (DFT)-based ab initio calculations of the spatial electron density distribution.

\section{Energy states of crystals from the In-Se system within the elementary energy bands concept}

It has been demonstrated [6-8] that one can obtain the reliable information about some peculiarities of chemical

*corresponding author; e-mail: sznajder@ur.edu.pl bonding in semiconducting crystals in the framework of the elementary energy band concept and the ab initio calculation of the spatial valence electron density distribution. It has been shown that there is a relation between the spatial valence electron density distribution in a unit cell and the so-called actual Wyckoff position where the maximum of this distribution is observed [6, 7]. At the same time, there is a relation between the site symmetry group of this position and the topology of the elementary energy bands which constitute the valence band of a semiconductor. It is known that in the case of the ionic binding the largest valence electron density is concentrated on the atoms, and some of them can coincide with the Wyckoff positions. Moreover, as demonstrated for a rhombic crystal [7], if none of the atoms in the unit cell is localized in the actual Wyckoff position, a covalent binding can be predicted to be dominant in such a crystal, when an additional requirement is fulfilled.

In order to select the actual Wyckoff position for the crystals from the In-Se system we use the empty lattice approximation, supplied with the most general information about crystal i.e., its space symmetry group, lattice parameters and the existence of the forbidden gap [6-9]. Next, we perform the DFT-based ab initio band structure calculations and calculations of the spatial valence electron density distributions for the crystals of the In-Se system. The obtained results confirm the predicted topology of the elementary energy bands constituting the respective valence bands, as well as the initially selected actual Wyckoff positions. It can be stated that a remarkable valence electron density is localized in these positions in the unit cells. The following actual Wyckoff positions can be attributed to the seven main compounds of the system i.e., $\operatorname{In}_{4} \mathrm{Se}_{3}\left(D_{2 h}^{12}\right)$ : $g(x, y, z)[10], \beta$-InSe $\left(D_{6 h}^{4}\right): k(x, 2 x, z), f(1 / 3,2 / 3, z)$, $d(1 / 3,2 / 3,3 / 4)$ [8], $\gamma-\operatorname{InSe}\left(C_{4 v}^{5}\right): a(\nu \boldsymbol{a}), b\left(\nu\left(\boldsymbol{a}_{1}+\boldsymbol{a}_{2}\right)+\right.$ $\left.\mu \boldsymbol{a}_{3}\right), \quad \varepsilon$-InSe $\left(D_{3 h}^{1}\right): \quad g(0,0, z), i(2 / 3,1 / 3, z), \alpha-\operatorname{In}_{2} \mathrm{Se}_{3}$ $\left(C_{6}^{6}\right): a(0,0, z), b(1 / 3,2 / 3, z), \gamma-\operatorname{In}_{2} \operatorname{Se}_{3}\left(C_{6}^{2}\right): a(x, y, z)$, 
$\operatorname{In}_{6} \mathrm{Se}_{7}\left(C_{2 h}^{2}\right): e\left(\alpha_{1} \boldsymbol{a}_{1}+\alpha_{2} \boldsymbol{a}_{2}\right)$. The actual Wyckoff positions for crystals from the In-Se system are mainly lines and planes which intersect the In-Se bonds. The calculation results show that the localization of a considerable spatial valence electron density between the respective pairs of atoms takes place in all crystals of the system (see Fig. 1). This indicates the dominance of the covalent type of bonding in the considered compounds.

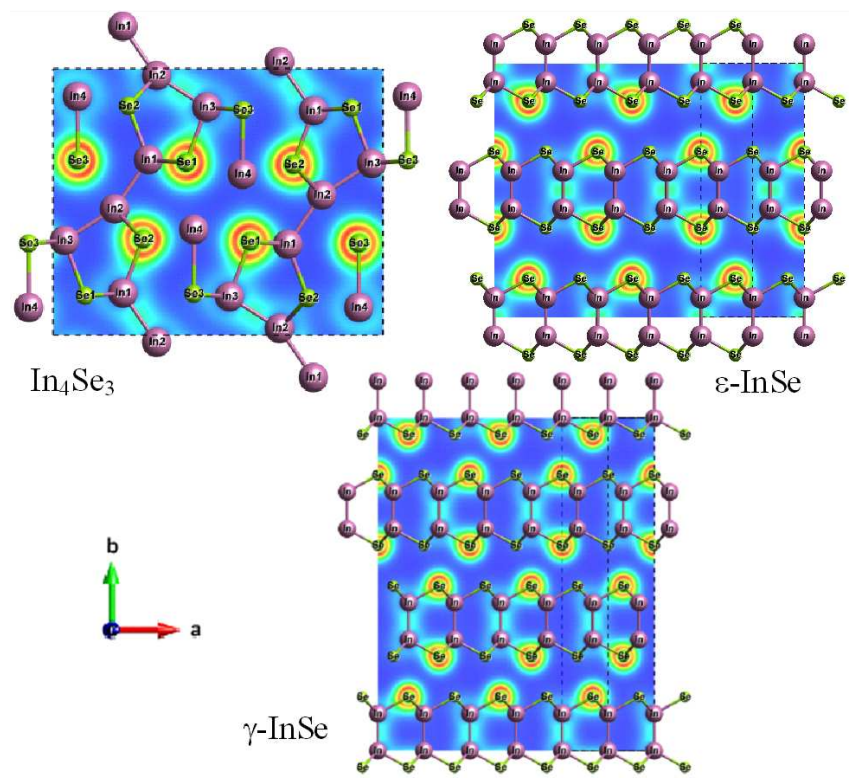

Fig. 1. Distributions of the spatial valence electron density in the primitive cells of $\operatorname{In}_{4} \mathrm{Se}_{3}$ (001), $\varepsilon$-InSe $(1 \overline{1} 0)$ and $\gamma$-InSe $(1 \overline{1} 0)$.

The results of our earlier calculations have shown that in the case of $\beta$-InSe and $\mathrm{In}_{4} \mathrm{Se}_{3}$ crystals, the inverse anisotropy of their chemical bonding in the real space is a main factor leading to the anomalous behavior of the dispersion law of charge carriers, which manifests itself as a low-energy nonparabolicity of the valence and conduction band extrema [10]. This peculiar shape of the dispersion law of charge carriers in $\mathrm{In}_{4} \mathrm{Se}_{3}$ is crucial for the creation of the localized electron condenson states that seem to be responsible for the enhanced thermoelectric properties of this semiconductor $[11,12]$. Our calculation results show that similar features of the dispersion laws occur in the band spectra of two further layered polytypes of InSe crystal, the $\varepsilon$-InSe (in the $\Gamma$ point) and $\gamma$-InSe (vicinity of A point) (Fig. 2). These features are related to the anomalous character of interlayer interaction present in these compounds i.e., the bonding between the In and Se atoms which belong to different layers is not of pre Van-der-Waals type but it has admixture of the covalent component. This peculiarity was also found for $\gamma$-InSe [13]. Let us note that to compare the band structures for different polytypes of the InSe crystal we have chosen the elementary supercell for $\gamma$-InSe, which consists of three atomic layers (12 atoms).

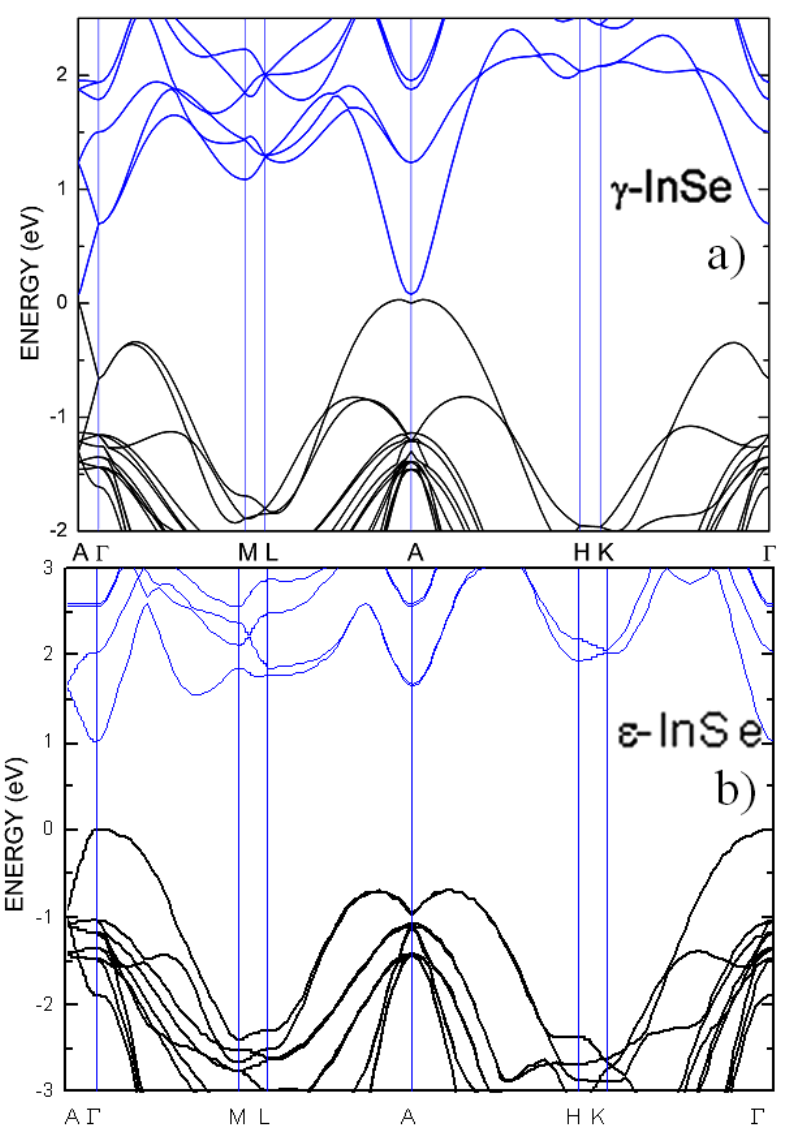

Fig. 2. Dispersion laws $E(\boldsymbol{k})$ in the vicinity of high symmetry points of the Brillouin zone for (a) $\gamma$-InSe and (b) $\varepsilon$-InSe.

\section{Mulliken's charges and the degree of ionicity in crystals of the In-Se system}

The Mulliken atomic charges resulting from the $a b i n i$ tio band structure calculations, performed employing the Abinit code, can be determined as a difference between the core charge and the total electron charge [14]:

$$
q_{A}=Z_{\mathrm{A}}-\sum_{i j \in A} P_{i j} S_{i j},
$$

where $Z_{A}$ is a charge of atomic core, $P_{i j}$ is the density matrix, $S_{i j}$ is an overlap matrix of the basis functions, $i$ and $j$ denote orbitals. The Mulliken charges are listed in Table I.

TABLE I

Mulliken's charges for indium selenide group.

\begin{tabular}{c|c|c}
\hline \hline Compound & $q_{\text {In }}$ & $q_{\mathrm{Se}}$ \\
\hline$\beta$-InSe & 0.522 & -0.522 \\
$\varepsilon$-InSe & $0.641,0.679$ & $-0.639,0.681$ \\
$\gamma$-InSe & $0.556,0.523$ & $-0.543,-0.535$ \\
\hline$\gamma-\mathrm{In}_{2} \mathrm{Se}_{3}$ & $0.897,0.773$ & $-0.532,-0.584,-0.554$ \\
\hline $\mathrm{In}_{4} \mathrm{Se}_{3}$ & $0.552,0.560,0.631,0.602$ & $-0.791,-0.805,-0.748$
\end{tabular}


As follows from Table II, the degree of ionization of each atom is not small. Indium atoms are the most ionized in the $\gamma-\mathrm{In}_{2} \mathrm{Se}_{3}$ polytype, hence this crystal has a remarkable degree of ionicity, similarly to the $\mathrm{In}_{4} \mathrm{Se}_{3}$ one. The Mullikan charges for In and Se atoms in the InSe polytypes are practically equal. The differences of $q_{\text {In }}$ for In atoms in the $\mathrm{In}_{2} \mathrm{Se}_{3}$ and $\mathrm{In}_{4} \mathrm{Se}_{3}$ crystals are the consequence of a different configurations of the atomic environment for indium. In order to calculate the degree of ionicity of these compounds that reflects the character of chemical binding, we use the empiric formula, proposed for the $\operatorname{In}_{6} \mathrm{Se}_{7}$ crystal [15]:

$$
f=\frac{1}{2}\left(1-\cos \left(\pi \frac{E_{\mathrm{AS}}}{E_{\mathrm{VB}}}\right)\right),
$$

where $E_{\mathrm{AS}}$ is the antisymmetric gap between the two lowest valence bands, and $E_{\mathrm{VB}}$ is the total valence band width. The values of $E_{\mathrm{AS}}$ and $E_{\mathrm{VB}}$ result from our $a b$ initio band structure calculations of the In-Se system.

TABLE II

The degree of ionicity for the compounds in the indium selenides group.

\begin{tabular}{c|c|c|c|c}
\hline \hline Compound & $E_{\mathrm{AS}}[\mathrm{eV}]$ & $E_{\mathrm{VB}}[\mathrm{eV}]$ & $f$ & $E_{\mathrm{g}}[\mathrm{eV}](\exp )$ \\
\hline$\beta$-InSe & 6.5 & 13.23 & 0.48 & 1.24 \\
$\varepsilon$-InSe & 6.98 & 15.14 & 0.44 & \\
$\gamma$-InSe & 6.97 & 13.88 & 0.50 & 1.35 \\
\hline$\alpha-\mathrm{In}_{2} \mathrm{Se}_{3}$ & 9 & 17 & 0.55 & 1.36 \\
$\gamma-\mathrm{In}_{2} \mathrm{Se}_{3}$ & 7.5 & 13.5 & 0.58 & 1.8 \\
\hline $\mathrm{In}_{4} \mathrm{Se}_{3}$ & 5.4 & 14 & 0.43 & 0.67 \\
\hline $\mathrm{In}_{6} \mathrm{Se}_{7}$ & 5.92 & 11.48 & 0.51 & 0.72
\end{tabular}

The largest degree of ionicity is obtained for the $\operatorname{In}_{2} \mathrm{Se}_{3}$ crystal which, in comparison with other indium selenides, has the largest forbidden energy gap.

\section{Conclusions}

Although the crystals from the In-Se system seem to be strongly covalent from the viewpoint of their crystal structure, a different degree of iconicity is found for them. Based upon the results of band structure calculation, the spatial valence electron density distribution, and the information about the actual Wyckoff positions found in the framework of the elementary energy bands concept, it can be concluded that crystals of the indium selenides group have the covalent character of the chemical binding with an ionic component.

\section{Acknowledgments}

M. Sznajder acknowledges the support from the Centre for Innovation and Transfer of Natural Sciences and Engineering Knowledge at the University of Rzeszów.

\section{References}

[1] J.-S. Rhyee, K.H. Lee, S.M. Lee, E. Cho, S.I. Kim, E. Lee, Y.S. Kwon, J.H. Shim, G. Kotliar, Nature 459, 965 (2009).

[2] J.H.C. Hogg, H.H. Sutherland, D.J. Williams, Chem. Commun. 23, 1568 (1971).

[3] I.I. Man, R.M. Imamov, S.A. Semiletov, Kristallografiya 21, 628 (1976).

[4] M. Ishikawa, T. Nakayama, Jpn. J. Appl. Phys. 36, L1576 (1997).

[5] J.H.C. Hogg, Acta Crystallogr. B27, 1630 (1971).

[6] D.M. Bercha, K.Z. Rushchanskii, M. Sznajder, A. Matkovskii, P. Potera, Phys. Rev. B 66, 195203 (2002).

[7] D.M. Bercha, I.V. Slipukhina, M. Sznajder, K.Z. Rushchanskii, Phys. Rev. B 70, 235206 (2004).

[8] K.E. Glukhov, N.K. Tovstyuk, Phys. Status Solidi B 247, 318 (2010).

[9] M. Sznajder, D.M. Bercha, K.E. Glukhov, I.V. Slipukhina, Acta Phys. Pol. A 110, 369 (2006).

[10] M. Sznajder, K.Z. Rushchanskii, L.Yu. Kharhkalis, D.M. Bercha, Phys. Status Solidi B 243, 592 (2006).

[11] Y.S. Lim, M. Jeong, W.-S. Seo, J.-H. Lee, C.-H. Park, M. Sznajder, L.Yu. Kharkhalis, D.M. Bercha, J. Yang, J. Phys. D Appl. Phys. 46, 275304 (2013).

[12] M. Jeong, Y.S. Lim, W.-S. Seo, J.-H. Lee, Ch.-H. Park, M. Sznajder, L.Yu. Kharkhalis, D.M. Bercha, J. Yang, J. Mater. Chem. A 1, 15342 (2013).

[13] P.G. de Costa, R.G. Dandrea, R.F. Wallis, M. Balkanskii, Phys. Rev. B 48, 14135 (1993).

[14] X. Gonze, J.-M. Beuken, R. Caracas, F. Detraux, M. Fuchs, G.-M. Rignanese, L. Sindic, M. Verstraete, G. Zerah, F. Jollet, M. Torrent, A. Roy, M. Mikami, Ph. Ghosez, J.-Y. Raty, D.C. Allan, Comp. Mater. Sci. B 25, 478 (2002); J.M. Soler, E. Artacho, J.D. Gale, A. García, J. Junquera, P. Ordejón, D. Sánchez-Portal, J. Phys. Condens. Matter 14, 2745 (2002).

[15] T.B. Nasr, H.B. Abdallah, R. Bennaceur, Physica B 405, 3427 (2010). 